\title{
A REDUCTION TECHNIQUE OF LARGE SCALE RCG INTERCONNECTS IN COMPLEX FREQUENCY DOMAIN
}

\author{
Y.Yamagami $\dagger$, Y.Nishio $\dagger$, A.Hattori†, A.Ushida $\dagger$ \\ $\dagger$ Department of Electrical and Electronic Engineering, \\ Tokushima University, Tokushima, 770-8506 JAPAN
}

\begin{abstract}
High frequency digital LSIs usually consist of many subcircuits coupled with multi-conductor interconnects embedded in the substrate. They sometimes cause serious problems of the fault switching operations due to the time-delays, crosstalks, reflections and so on. In order to solve these problems, it is very important to develop a user-friendly simulator for the analysis of LSIs coupled with interconnects. At the reduction algorithm, we first calculate the dominant poles which give the large effects to the transient response, and the corresponding residues are estimated by the least squares method. Thus, the interconnect is replaced by the equivalent circuit realizing the partial fractions.
\end{abstract}

\section{Introduction}

The analysis of high speed LSI chips is becoming more and more important for their designing. The chips are usually coupled with interconnects embedded in the substrate, and interconnects sometimes cause the fault switching of chips due to the signal delays, crosstalks and so on [1]-[8]. The Elmore resistance-capacitance ( $\mathrm{RC}$ ) delay metric is popular due to its simple closed-form expression, computation speed and fidelity with respect to the simulation [3]. The closedform combining the delay and crosstalk is firstly obtained in the reference [4]. Improved techniques [5]-[8] are also proposed later for improving the accuracy and the practical applications in the simulations.

Nowadays, AWE(asymptotic waveform evaluation)[9] is widely used as a reduction technique of large scale networks coupled with interconnects, whose algorithm is based on the moment-matching method and Padè approximation. Unfortunately, one of the serious problems of the momentmatching method is that the poles located far from the origin sometimes become erroneous, because the admittance or impedance matrix via AWE is described in the form of power series with the complex frequency $s$ based on the Maclaurin expansion. To overcome the problem, Nakhla et al. have proposed CFH (complex frequency hopping) [10] for calculating the exact poles. The algorithm can find out the exact poles by properly hopping the origin of Taylor expansion on the complex axis. The other is based on a multi-point Padè approximation [10]. Both of them need properly to choose some points in the complex frequency domain to obtain the exact Taylor series and Padè approximation. PRIMA (passive reduced-order interconnect macromodeling algorithm) [11] is another reduction algorithm which is an extension of the block Arnoldi technique to include guaranteed passivity. 0-7803-7762-1/03/\$17.00 @2003 IEEE
In order to apply this algorithm to the circuits with interconnects, we need two steps such that the interconnect is modeled by a finite order system, and afterward, Arnoldibased congruence transform is applied to the system to form its reduced order model.

In this paper, we consider LSIs such that large scale gatearray circuits are coupled with interconnects embedded in the substrate. In this case, the capacitance component of the interconnect is dominant compared to the inductance, and the diffusion resistance is very large compared to those of PCBs [2], so that we assume the interconnect as RCG interconnect instead of RLCG in this paper. We first derive the impedance matrix at the near and far end ports. We prove that all the poles of the impedance matrix are located on the negative real axis in the complex plane. The residues are decided by the least-squares method in such manner that the response curves coincide with those from the impedance matrix in the complex frequency domain of interest. We found from the simulation results that the impedance matrix can be approximately described by the partial fractions with the few poles located near the origin. The reduction rate is very large especially for a large scale interconnect. Hence, each element of the impedance matrix can be described by a series of partial fractions, and the interconnect is easily synthesized by the asymptotic equivalent circuit satisfying the partial fractions. Thus, we can easily develop SPICEoriented simulator of LSIs coupled with RCG interconnects.

\section{Poles and residues of the impedance matrix}

Now, consider a uniform $N$ coupled RCG interconnect. Assume the telegraph equation can be described by

$$
\frac{d \mathbf{V}(x, s)}{d x}=-\mathbf{R I}(x, s), \quad \frac{d \mathbf{I}(x, s)}{d x}=-(\mathbf{G}+s \mathbf{C}) \mathbf{V}(x, s)
$$

in the complex frequency domain, where $\mathbf{R}, \mathbf{C}$ and $\mathbf{G}$ are positive definite symmetric matrices. Let us introduce the matrices $\mathbf{P}_{v}(s)$ and $\mathbf{P}_{c}(s)$ to transform them into the diagonal forms. Thus, we have

$$
\left.\begin{array}{rl}
\operatorname{diag}\left[\lambda_{i}(s)^{2}\right] & =\mathbf{P}_{v}(s)^{-1} \mathbf{R}(\mathbf{G}+s \mathbf{C}) \mathbf{P}_{v}(s) \\
\operatorname{diag}\left[\lambda_{i}(s)^{2}\right] & =\mathbf{P}_{c}(s)^{-1}(\mathbf{G}+s \mathbf{C}) \mathbf{R} \mathbf{P}_{c}(s)
\end{array}\right\}
$$

where we can choose one of $\mathbf{P}_{c}(s)$ 's in the following relations:

$$
\left.\begin{array}{l}
\mathbf{P}_{c}(s)=\mathbf{R}^{-1} \mathbf{P}_{v}(s) \boldsymbol{\Gamma}(s), \\
\mathbf{P}_{c}(s)=(\mathbf{G}+s \mathbf{C}) \mathbf{P}_{v}(s) \boldsymbol{\Gamma}(s)^{-1}, \\
\mathbf{P}_{c}(s)^{-1}=\mathbf{P}_{v}(s)^{T}
\end{array}\right\}
$$


for $\boldsymbol{\Gamma}(s)=\operatorname{diag}\left[\lambda_{i}(s)\right]$. Then, the input and output relations at the near and far ends are described by the impedance matrix as follows:

$$
\left[\begin{array}{l}
\mathbf{V}(0, s) \\
\mathbf{V}(d, s)
\end{array}\right]=\left[\begin{array}{ll}
\mathbf{Z}_{11}(s) & \mathbf{Z}_{12}(s) \\
\mathbf{Z}_{21}(s) & \mathbf{Z}_{22}(s)
\end{array}\right]\left[\begin{array}{c}
\mathbf{I}(0, s) \\
-\mathbf{I}(d, s)
\end{array}\right]
$$

where

$$
\begin{aligned}
& \mathbf{Z}_{11}(s)=\mathbf{Z}_{22}(s)=\mathbf{P}_{v}(s) \operatorname{diag}\left[\operatorname{coth} \lambda_{i}(s) d\right] \mathbf{P}_{c}(s)^{-1} \\
& \mathbf{Z}_{12}(s)=\mathbf{Z}_{21}(s)=\mathbf{P}_{v}(s) \operatorname{diag}\left[\sinh \lambda_{i}(s) d\right]^{-1} \mathbf{P}_{c}(s)^{-1}
\end{aligned}
$$

Observe that all the poles of impedance matrix are found at the locations satisfying $\sinh \lambda_{i}(s) d=0$. Thus, we have the following theorem for the calculation of poles.

Theorem 1: The locations of poles satisfying relations (5) are found by solving the following equation:

$$
\left|\mathbf{R}(\mathbf{G}+s \mathbf{C})+\left(\frac{n \pi}{d}\right)^{2} \mathbf{I}\right|=0, \quad n=0,1,2, \ldots
$$

where $d$ is the length of the interconnect.

Proof In the case of $n \neq 0$, we have from (5) that the poles satisfy the following relation:

$$
\left|\mathbf{P}_{c}(s) \operatorname{diag}\left[\sinh \lambda_{i}(s) d\right] \mathbf{P}_{v}(s)^{-1}\right|=0
$$

and

$$
\left|\mathbf{P}_{c}(s) \operatorname{diag}\left[\tanh \lambda_{i}(s) d\right] \mathbf{P}_{v}(s)^{-1}\right|=0
$$

Since $\mathbf{P}_{v}(s)$ and $\mathbf{P}_{c}(s)$ are nonsingular for the nonzero eigenvalues, the poles satisfying the above two relations are given by

$$
\sinh \lambda_{i}(s) d=0, \quad i=1,2, \ldots, N
$$

where $N$ shows a number of the multi-conductors. Namely, we have

$$
\lambda_{i}(s) d=j n \pi, \quad i=1,2, \ldots, N, \quad n=1,2, \ldots
$$

Therefore, the characteristic equation obtained from (2) needs to satisfy the relations (6). On the other hand, we have from (2) that the zero eigenvalue satisfies $|\mathbf{G}+s \mathbf{C}|=0$, which corresponds to $n=0$ in (6).

Q.E.D.

Theorem 2: Let $\mathbf{R}, \mathbf{C}$ and $\mathbf{G}$ be positive definite symmetric matrices. Then, all the poles of $R C G$ interconnect are located on the negative real axis.

Proof The poles satisfying relation (6) are given by

$$
\left|(\mathbf{G}+s \mathbf{C})+\left(\frac{n \pi}{d}\right)^{2} \mathbf{R}^{-1}\right|=0, \quad n=0,1,2, \ldots
$$

For simplicity, we will rewrite the matrix given by (11) as follows:

$$
\left|s \mathbf{C}+\mathbf{H}_{n}\right|=0, \quad \text { where } \mathbf{H}_{n}=\left(\frac{n \pi}{d}\right)^{2} \mathbf{R}^{-1}+\mathbf{G}
$$

Observe that $\mathbf{H}_{n}$ is still a positive definite symmetric matrix. Thus, it can be transformed into the following diagonal form:

$$
\mathbf{S Q}^{T}\left(\mathbf{H}_{n}+s \mathbf{C}\right) \mathbf{Q S}=\mathbf{S Q}^{T} \mathbf{H}_{n} \mathbf{Q S}+s \mathbf{I}=\mathbf{0}
$$

with an orthogonal matrix $\mathbf{Q}$ and $\mathbf{S}=\operatorname{diag}\left[\mathrm{C}_{\mathrm{ii}}\right]^{-\frac{1}{2}}$, where $C_{i i}, i=1, \ldots, N$ are the eigenvalues of $\mathbf{C}$. Here, $\mathbf{S Q}^{T} \mathbf{H}_{n} \mathbf{Q S}$ is a positive real symmetric matrix, so that all the poles satisfying (13) are negative real numbers.

Q.E.D.

Now, consider the numerical technique for calculating the poles satisfying (6). Let us rewrite (11) in the following form;

$$
|s \mathbf{I}-\mathbf{A}|=0
$$

where

$$
\mathbf{A}=-(\mathbf{R C})^{-1}\left(\left(\frac{n \pi}{d}\right)^{2} \mathbf{I}+\mathbf{R G}\right)
$$

The relation (14) corresponds to the characteristic equation of a matrix A. Then, we can apply the Bocher formula as follows:

Bocher formular [13]: For an $n \times n$ matrix $\mathbf{A}$, set

$$
|s \mathbf{I}-\mathbf{A}|=\alpha_{0}+s \alpha_{1}+\cdots+s^{n-1} \alpha_{n-1}+s^{n}=0
$$

Then, $\alpha_{0}, \alpha_{1}, \ldots, \alpha_{n-1}$ are given by

$$
\begin{aligned}
& \alpha_{n-1}=-\operatorname{trace}(\mathbf{A}) \\
& \alpha_{n-2}=-\frac{1}{2}\left[\alpha_{n-1} \operatorname{trace}(\mathbf{A})+\operatorname{trace}\left(\mathbf{A}^{2}\right)\right] \\
& \alpha_{0}=-\frac{1}{n}\left[\alpha_{1} \operatorname{trace}(\mathbf{A})+\alpha_{2} \operatorname{trace}\left(\mathbf{A}^{2}\right)+\cdots\right. \\
& \left.\left.+\alpha_{n-1} \operatorname{trace}\left(\mathbf{A}^{n-1}\right)+\operatorname{trace}\left(\mathbf{A}^{n}\right)\right]\right\}
\end{aligned}
$$

Thus, the poles satisfying algebraic equation (16) can be calculated by the application of standard numerical methods for solving the algebraic equation such as Bairstow algorithm.

Now, if we can estimate the residues corresponding to the poles, the impedance matrix given by (5) can be described by the partial fractions as follows:

$Z_{11, i j}(s)=Z_{22, i j}(s)=\frac{k_{0, i j}}{s+p_{0}}+\frac{k_{1, i j}}{s+p_{1}}+\frac{k_{2, i j}}{s+p_{2}}+\frac{k_{3, i j}}{s+p_{3}}+\cdots$

$Z_{12, i j}(s)=Z_{21, i j}(s)=\frac{k_{0, i j}}{s+p_{0}}-\frac{k_{1, i j}}{s+p_{1}}+\frac{k_{2, i j}}{s+p_{2}}-\frac{k_{3, i j}}{s+p_{3}}+\cdots$

for $i, j=1,2, \ldots, N$. The residues can be calculated by the least squares method in such manner that the frequency responses of (18) are well approximated by those of the impedance matrices (5).

\section{Large scale RCG interconnects}

Now, let us discuss the reduction algorithm of a large scale RCG interconnect and the asymptotic equivalent circuit model. To understand our algorithm, we consider an example of $N$ coupled interconnect whose parameters are given as follows:

$$
\left[\begin{array}{c}
R_{i, i}=5 \Omega / \mu \mathrm{m}, R_{i, i-1}=R_{i, i+1}=1 \Omega / \mu \mathrm{m}, \\
R_{i, i-2}=R_{i, i+2}=0.1 \Omega / \mu \mathrm{m}, \\
i=1,2, \ldots, N, \text { Other elements of } \mathbf{R} \text { are zeros }
\end{array}\right]
$$

$$
\left[\begin{array}{c}
G_{i, i}=10 \mathrm{mS} / \mu \mathrm{m}, G_{i, i-1}=G_{i, i+1}=-1 \mathrm{mS} / \mu \mathrm{m}, \\
G_{i, i-2}=G_{i, i+2}=-0.1 \mathrm{mS} / \mu \mathrm{m} \\
i=1,2, \ldots, N, \text { Other elements of } \mathbf{G} \text { are zeros }
\end{array}\right]
$$

$$
\left[\begin{array}{c}
C_{i, i}=6.28 \mathrm{fF} / \mu \mathrm{m}, C_{i, i-1}=C_{i, i+1}=-0.49 \mathrm{fF} / \mu \mathrm{m}, \\
C_{i, i-2}=C_{i, i+2}=-0.03 \mathrm{fF} / \mu \mathrm{m} \\
i=1,2, \ldots, N, \text { Other elements of } \mathbf{C} \text { are zeros }
\end{array}\right]
$$


We also assume the length $d=5 \mu \mathrm{m}$. We first calculate the poles using the relation (6), and have gotten the tables $3.1,3.2$ and 3.3, where we have neglected the poles with the value less than -400 , because the poles far from the origin will have only small effects to the transient response.

Table 3.1 Poles for $N=1\left(\left|p_{\max }\right|<400\right)$
\begin{tabular}{|c|c|c|c|c|c|c|}
\hline No. & $\mathrm{n}=0$ & $\mathrm{n}=1$ & $\mathrm{n}=2$ & $\mathrm{n}=3$ & $\mathrm{n}=4$ & $\mathrm{n}=5$ \\
\hline 1 & -1.592 & -14.17 & -51.88 & -114.8 & -202.8 & -315.9 \\
\hline
\end{tabular}

Table 3.2 Poles for $N=2\left(\left|p_{\max }\right|<400\right)$

\begin{tabular}{|c|c|c|c|c|c|c|}
\hline No. & $\mathrm{n}=0$ & $\mathrm{n}=1$ & $\mathrm{n}=2$ & $\mathrm{n}=3$ & $\mathrm{n}=4$ & $\mathrm{n}=5$ \\
\hline 1 & -1.554 & -12.92 & -47.01 & -103.8 & -183.4 & -285.7 \\
2 & -1.625 & -16.20 & -59.94 & -132.8 & -234.9 & -366.1 \\
\hline
\end{tabular}

Table 3.3 Poles for $N=10\left(\left|p_{\max }\right|<400\right)$

\begin{tabular}{|c|c|c|c|c|c|c|}
\hline No. & $\mathrm{n}=0$ & $\mathrm{n}=1$ & $\mathrm{n}=2$ & $\mathrm{n}=3$ & $\mathrm{n}=4$ & $\mathrm{n}=5$ \\
\hline 1 & -1.496 & -12.02 & -43.61 & -96.25 & -169.9 & -256.5 \\
2 & -1.515 & -12.25 & -44.45 & -98.12 & -173.3 & -261.4 \\
3 & -1.542 & -12.65 & -45.96 & -101.5 & -179.2 & -279.2 \\
4 & -1.570 & -13.24 & -48.25 & -106.6 & -188.3 & -293.3 \\
5 & -1.595 & -14.04 & -51.36 & -113.6 & -200.7 & -312.6 \\
6 & -1.615 & -15.01 & -55.18 & -122.1 & -215.9 & -336.4 \\
7 & -1.628 & -16.08 & -59.42 & -131.7 & -232.8 & -362.9 \\
8 & -1.635 & -17.12 & -63.58 & -141.0 & -249.4 & -388.8 \\
9 & -1.638 & -18.00 & -67.07 & -148.9 & -263.4 & -394.9 \\
10 & -1.639 & -18.57 & -69.38 & -154.1 & -272.6 & -425.0 \\
\hline
\end{tabular}

where $N=1,2,10$ means single line, 2-coupled and 10coupled interconnects, respectively. It is remarkable that the number of poles for $N$-coupled interconnect is given by $N \times(M+1)$ if we consider up to $n=0,1, \ldots, M$ in (6). Therefore, the number rapidly increases as $M$ and $N$, and the resulting asymptotic equivalent circuit of the interconnect will become very complicate and large scale network if we consider all the $N \times(M+1)$ poles.

We found from the tables that the poles for $N=1$ are always located around the center position for every $n$th pole group; i.e. between No.1 and No.2 for $N=2$ as shown by the table 3.2, and between No.5 and No.6 for $N=10$ as shown by the table 3.3 , respectively. Hence, we always assume the 6 poles of $N=1$ given by the table 3.1 in our reduction algorithm as the approximate representative poles in all the cases of $N$ even if $N=100,1000$ or more.

In this case, each element given by (5) can be written with the 6 terms in the following form;

$$
\begin{gathered}
Z_{k l, i j}=\frac{k_{1, k l, i j}}{s+1.592}+\frac{k_{2, k l, i j}}{s+14.17}+\frac{k_{3, k l, i j}}{s+51.88}+\frac{k_{4, k l, i j}}{s+114.8} \\
+\frac{k_{5, k l, i j}}{s+202.8}+\frac{k_{6, k l, i j}}{s+315.9}
\end{gathered}
$$

for $k, l=1,2, \quad i, j=1,2, \ldots, N$.

Now, let us calculate the residues $k_{1, k l, i j}, \ldots, k_{6, k l, i j}$ by the application of the least squares method, in such a manner that the errors between the frequency response curve from the impedance matrix given by (5) and the response curve satisfying (20) become the smallest in the frequency of interesting. The frequency response curves using the 6 poles are shown in Fig.1 (a) and (b). They are good agreement in each other. We also found from the numerical results that the value of residues far from the diagonal elements in $\mathbf{Z}_{i j}, i, j=1,2$ become smaller and smaller.
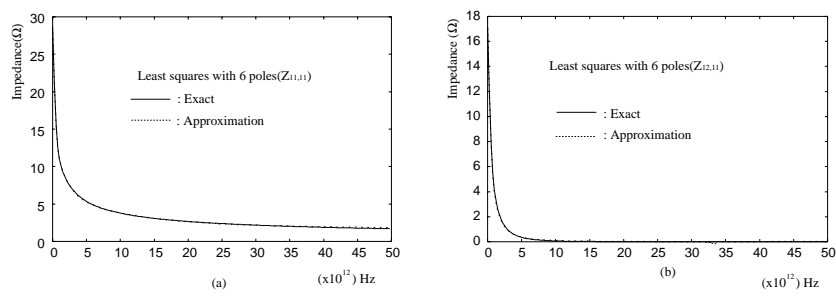

Fig.1: Least squares method with 6 poles (a) $Z_{11,11}$, (b) $Z_{12,11}$

Therefore, it is enough to choose only few elements around the diagonal element in each row as the dominant elements. Hence, each sub-matrix in (5) can be written as follows:

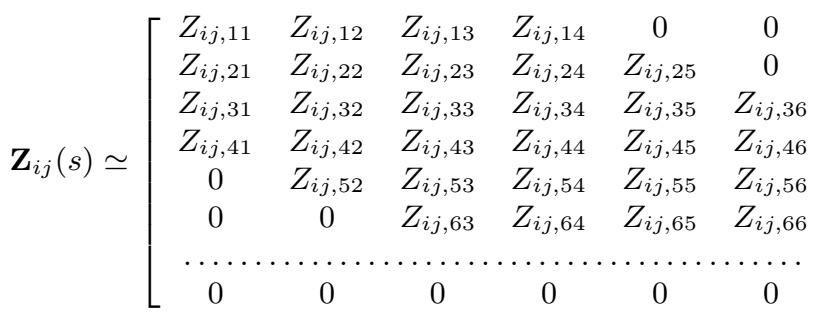

$$
\begin{aligned}
& \left.\begin{array}{ccccc}
0 & 0 & 0 & 0 & 0 \\
0 & 0 & 0 & 0 & 0 \\
0 & 0 & 0 & 0 & 0 \\
Z_{i j, 47} & 0 & 0 & 0 & 0 \\
Z_{i j, 57} & Z_{i j, 58} & 0 & 0 & 0 \\
Z_{i j, 67} & Z_{i j, 68} & Z_{i j, 69} & 0 & 0 \\
\ldots \ldots \ldots \ldots \ldots \ldots \ldots \ldots \ldots \ldots \ldots \ldots \ldots \ldots \ldots \ldots \ldots \ldots \ldots & \ldots \ldots \ldots
\end{array}\right]
\end{aligned}
$$

for $i, j=1,2$. Furthermore, we have approximately the following relations:

$$
\left[\begin{array}{c}
Z_{i j, 11} \approx \cdots \approx Z_{i j, N N}, \text { for diagonal elements } \\
Z_{i j, k k+1} \approx Z_{i j, k k-1}, Z_{i j, k k+2} \approx Z_{i j, k k-2}, \\
Z_{i j, k k+3} \approx Z_{i j, k k-3}, \text { for nonzeo kth terms }
\end{array}\right]
$$

Now, consider the asymptotic equivalent circuit model of (4) as shown in Fig.2.1(a). Using the relations (22), the terminal voltages at the near and far ends can be written as follows:

$$
\begin{aligned}
& V_{k}(0, s) \approx Z_{11, k k} I_{k}(0, s) \\
&+Z_{11, k k+1}\left(I_{k-1}(0, s)+I_{k+1}(0, s)\right) \\
&+Z_{11, k k+2}\left(I_{k-2}(0, s)+I_{k+2}(0, s)\right) \\
&+Z_{11, k k+3}\left(I_{k-3}(0, s)+I_{k+3}(0, s)\right) \\
&+Z_{12, k k} I_{k}(d, s) \\
&+Z_{12, k k+1}\left(I_{k-1}(d, s)+I_{k+1}(d, s)\right) \\
&+Z_{12, k k+2}\left(I_{k-2}(d, s)+I_{k+2}(d, s)\right) \\
&+Z_{12, k k+3}\left(I_{k-3}(d, s)+I_{k+3}(d, s)\right) \\
& \\
& V_{k}\left(d, k k I_{k}(0, s)\right. \\
&+ \\
&+Z_{12, k k+1}\left(I_{k-1}(0, s)+I_{k+1}(0, s)\right) \\
&+Z_{12, k k+2}\left(I_{k-2}(0, s)+I_{k+2}(0, s)\right) \\
&+Z_{12, k k+3}\left(I_{k-3}(0, s)+I_{k+3}(0, s)\right) \\
&+Z_{11, k k} I_{k}(d, s) \\
&+Z_{11, k k+1}\left(I_{k-1}(d, s)+I_{k+1}(d, s)\right) \\
&+Z_{11, k k+2}\left(I_{k-2}(d, s)+I_{k+2}(d, s)\right) \\
&+Z_{11, k k+3}\left(I_{k-3}(d, s)+I_{k+3}(d, s)\right)
\end{aligned}
$$

$k=1,2, \ldots, N Z_{11, k k}$ in the first term of (23.1) and the 5 th term of (23.2) correspond to the self impedance $Z_{11}, Z_{22}, \ldots, Z_{N N}$ shown in Fig.2.1 (b) and Fig.2.2(b). Other terms consist of the current controlled voltage sources as shown Fig.2.2(a). 


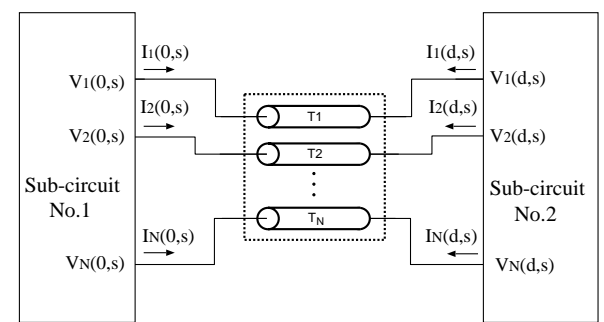

(a) Multi-conductor interconnect

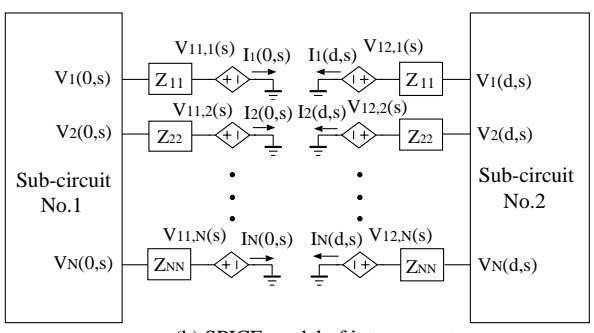

(b) SPICE model of interconnect

Fig.2.1: SPICE model of multi-conductor interconnect

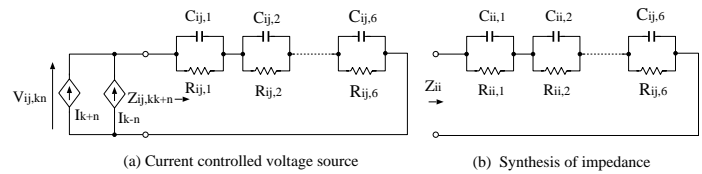

Fig.2.2: (a) Current controlled voltage source, (b) Self-impedance

\section{Illustrative examples}

Consider a fulladder circuit coupled with interconnects as shown in Fig.3(a).

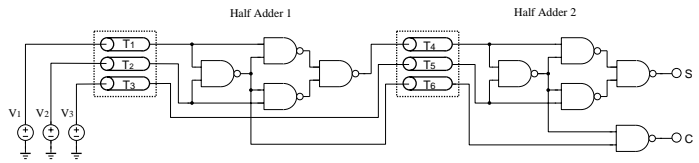

Fig.3(a): Fulladder coupled with interconnects $d=20 \mu \mathrm{m}$

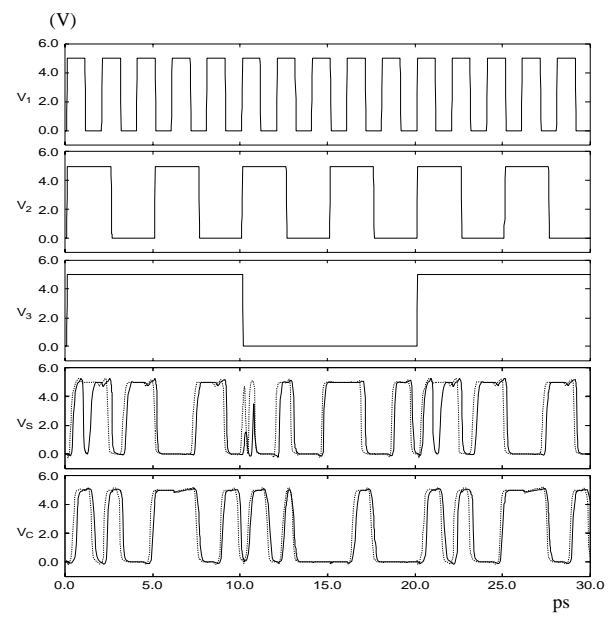

Fig.3(b): Transient response of the fulladder circuit.

The solid lines show the responses with interconnects, and the dotted lines the responses without the interconnects

The transient responses at the output stages are shown by solid lines in Fig.3(b), where the dotted lines show the re- sponses without the interconnects. Observe that the response coupled with interconnects has the complicated response due to the time-delays, crosstalks, reflections and so on.

\section{Conclusions and remarks}

We proposed a reduction algorithm for large scale RCG interconnects, where they are replaced by simple asymptotic equivalent circuits with current controlled voltage sources. The reduction rate will be very large especially for the large scale interconnects. Therefore, we can easily get the transient responses with the SPICE simulator. The algorithm will be efficiently applied to the large scale circuits such as gate-arrays coupled with interconnects in the substrate.

\section{REFERENCES}

[1] M.Celik, L.Pileggi and A.Odabasioglu, IC Interconnect Analysis, Kluwer Academic Pub., 2002.

[2] J.L.Wyatt,Jr., "Signal propergation delay in RC models for interconnect," Circuit Analysis, Simulation and Design, A.E.Ruehli(Editor), Elsevier Science Pub. B.V.(North-Holland), pp.254-291, 1987.

[3] W.C.Elmore, "The transient response of damped linear networks with particular regard to wideband amplifiers, " J. Appl. Phys., vol.19, pp.55-63, Jan. 1948.

[4] T.Sakurai, "Closed-form expressions for interconnection delay, coupling, and crosstalk in VLSI's," IEEE Trans. Electron Devices, vol.40, pp.118-124, 1991.

[5] S-Y.Kim, N.Gopal and L.T.Pillage, "Time-domain macromodels for VLSI interconnect analysis," IEEE Trans. Computer-Aided Design, vol.13, pp.1257-1270, 1994.

[6] R.Gupta, B.Tutuianu and L.T.Pileggi, "The Elmore delay as a bound for RC trees with generalized input signals," IEEE Trans. Computer-Aided Design, vol.16, pp.95-104, 1997.

[7] C.J.Alpert, A.Devgan and C.V.Kashap, "RC delay metrics for performance optimization," IEEE Trans. Computer-Aided Design, vol.20, pp.571-582, 2001.

[8] T.Kimura and M.Okumura, "An efficient reduction method of a substrate RC network model," IEICE Trans. Fundamentals, vol.E84-A, pp.698-704, 2001.

[9] E.Chiprout and M.S.Nakhla, Asymptotic Waveform Evaluation and Moment Matching for Interconnect Analysis, Kluwer Academic Pub., 1994.

[10] M.Celik, O.Ocali, M.A.Tan and A.Atalar, "Pole-zero computation in microwave circuits using multipoint Padè approximation," IEEE Trans. Circuits and Systems, vol.42, pp.6-13, 1995.

[11] A.Odabasioglu, M.Celik and L.T.Pileggi, "PRIMA: Passive reduced-order interconnect macromodeling algorithm," IEEE Trans. Computer Aided Design, vol.17, pp.645-654, 1998.

[12] A.Ushida, K.Urabe, Y.Yamagami and Y.Nishio, "Asymptotic equivalent circuits of interconnects based on complex frequency method," ECCTD'01, vol.3, pp.29-32, 2001.

[13] M.Bocher, Introduction to Higher Algebra, Dover, New York, 1964. 